\title{
A SUFFICIENCY PROOF FOR ISOPERIMETRIC PROBLEMS IN THE CALCULUS OF VARIATIONS
}

\section{R. HESTENES}

The purpose of the present paper is to show that the sufficiency theorems for a strong relative minimum for isoperimetric problems can be obtained from those for simple integral problems with only a little additional argument. The method here used is a simple extension of one used by Birkhoff and Hestenes* for a special isoperimetric problem. Heretofore sufficiency theorems of this type have been obtained from those for a restricted relative minimum by the application of the theorem of Lindeberg. Sufficiency theorems for a restricted relative minimum can be obtained either from the theories of the problems of Lagrange and Bolza or by an argument analogous to that used for simple integrals.

The problem to be considered is that of minimizing an integral

$$
J=\int_{x_{1}}^{x_{2}} f\left(x, y, y^{\prime}\right) d x=\int_{x_{1}}^{x_{2}} f\left(x, y_{1}, \cdots, y_{n}, y_{1}^{\prime}, \cdots, y_{n}^{\prime}\right) d x
$$

in a class of admissible arcs

$$
y_{i}(x), \quad x_{1} \leqq x \leqq x_{2} ; i=1, \cdots, n,
$$

joining two fixed points 1 and 2 and satisfying a set of isoperimetric conditions

$$
J_{\alpha}=\int_{x_{1}}^{x_{2}} f_{\alpha}\left(x, y, y^{\prime}\right) d x=l_{\alpha}, \quad \alpha=1, \cdots, m,
$$

where the $l$ 's are constants. It will be assumed that the functions $f, f_{\alpha}$ are defined and have continuous derivatives of the first three orders in a region $R$ of points $\left(x, y, y^{\prime}\right)$. The points of $R$ will be called $a d$ missible. A continuous arc (1) that can be divided into a finite number of subarcs on each of which it has continuous derivatives will be called admissible if its elements $\left(x, y, y^{\prime}\right)$ are all admissible.

Associated with the problem is an integral of the form

$$
I_{\lambda}=\int_{x_{1}}^{x_{2}} F\left(x, y, y^{\prime}, \lambda\right) d x,
$$

* Natural isoperimetric conditions in the calculus of variations, Duke Mathematical Journal, vol. 1 (1935), pp. 251-258. The method used in this paper was suggested by Professor Birkhoff. 
where $F=f+\lambda_{\alpha} f_{\alpha}{ }^{*}$ and the $\lambda$ 's are constant multipliers. It is in terms of this integral that the sufficiency conditions stated below are given. An admissible arc (1) and a set of constants $\lambda_{\alpha}$ having continuous second derivatives will be said to form an isoperimetric extremal if they satisfy the Euler-Lagrange equations

$$
F_{y_{i}}-\frac{d F_{y_{i}^{\prime}}}{d x}=0
$$

Let $E_{0}$ be an isoperimetric extremal joining the points 1 and 2 and satisfying the conditions (2). It will be assumed that $E_{0}$ is normal, that is, that the equations $P_{i \alpha} a_{\alpha}=0$, where

$$
P_{i \alpha}=f_{\alpha y_{i}}-\frac{d f_{\alpha y_{i}}}{d x}
$$

hold along $E_{0}$ only in case the constants $a_{\alpha}$ are all zero. Subarcs of $E_{0}$ need not be normal. We shall suppose that the extremal $E_{0}$ has the following further properties. At each element $\left(x, y, y^{\prime}, \lambda\right)$ in a neighborhood of those belonging to $E_{0}$ the inequality

$$
F\left(x, y, Y^{\prime}, \lambda\right)-F\left(x, y, y^{\prime}, \lambda\right)-\left(Y_{i}^{\prime}-y_{i}^{\prime}\right) F_{y_{i^{\prime}}}\left(x, y, y^{\prime}, \lambda\right)>0
$$

holds for every admissible set $\left(x, y, Y^{\prime}\right) \neq\left(x, y, y^{\prime}\right)$. Moreover along $E_{0}$ the inequality $F_{y^{\prime} y_{k}} \pi_{i} \pi_{k}>0$ holds for every set $(\pi) \neq(0)$. Finally

$$
Q(\eta)=\int_{x_{1}}^{x_{2}} 2 \omega\left(x, \eta, \eta^{\prime}\right) d x
$$

the second variation of $I_{\lambda}$ along $E_{0}$, is positive for every non-null set of admissible variations $\eta_{i}(x),\left(x_{1} \leqq x \leqq x_{2}\right)$, vanishing at $x=x_{1}$ and $x=x_{2}$ and satisfying with $E_{0}$ the equations

$$
L_{\alpha}(\eta)=\int_{x_{1}}^{x_{2}}\left\{f_{\alpha y_{i} \eta_{i}}+f_{\alpha y_{i}{ }^{\prime} \eta_{i}^{\prime}}\right\} d x=0 .
$$

An admissible variation $\eta_{i}(x),\left(x_{1} \leqq x \leqq x_{2}\right)$, is an arc having continuity properties like those of admissible arcs. The integrand $2 \omega$ appearing in $Q(\eta)$ is of the form $2 \omega=F_{y_{i} y_{k}} \eta_{i} \eta_{k}+2 F_{y_{i} y_{k^{\prime}}{ }_{i}} \eta_{k}^{\prime}+F_{y_{i}{ }^{\prime} y_{k}} \eta_{i}^{\prime} \eta_{k}^{\prime}$.

The theorem to be proved is the following:

THEOREM. If $E_{0}$ has the properties described above, there is a neighborhood $\mathcal{F}$ of $E_{0}$ in $x y$-space such that the inequality $J(C)>J\left(E_{0}\right)$ holds for every admissible arc $C$ in 7 joining 1 and 2, satisfying conditions (2), and not identical with $E_{0}$.

\footnotetext{
* Repeated indices denote summation.
} 
The proof of this result is based on two lemmas to be given below. In these lemmas we use the following definition of conjugate points. A value $x_{3} \neq x_{1}$ is said to define a point 3 conjugate to 1 on $E_{0}$ relative to $I_{\lambda}$ if there is a solution $\eta_{i}(x)$ of the equations

$$
K_{i}(\eta) \equiv \omega_{\eta_{i}}-\frac{d \omega_{\eta_{i}{ }^{\prime}}}{d x}=0
$$

having continuous second derivatives and vanishing at $x_{1}$ and $x_{3}$ but not identically zero on $x_{1} x_{3}$.

LEMma 1. If $E_{0}$ has on it no point 3 conjugate to 1 relative to $I_{\lambda}$, there exist neighborhoods $\mathcal{F}$ of $E_{0}$ in $x y$-space, $N$ of the end values of $E_{0}$ in $\left(x_{1} y_{1} x_{2} y_{2}\right)$-space, and $\Lambda$ of the multipliers $\lambda_{\alpha}$ belonging to $E_{0}$ such that for every set $\left(x_{1} y_{1} x_{2} y_{2}\right)$ in $N$ and $\lambda_{\alpha}$ in $\Lambda$ there is an isoperimetric extremal $E_{\lambda}$ in 7 having $\left(x_{1} y_{1} x_{2} y_{2}\right)$ as its end values and $\lambda_{\alpha}$ as its multipliers. Moreover for these values of $\lambda_{\alpha}$ the inequality $I_{\lambda}(C)>I_{\lambda}\left(E_{\lambda}\right)$ holds for every admissible arc $C$ in $\mathcal{F}$ joining the ends of $E_{\lambda}$ and not identical with $E_{\lambda}$.

This lemma is analogous to one given by Hahn and has been established by Birkhoff and Hestenes* following a method given by Bliss $\dagger$ in the proof of a similar theorem for the problem of Bolza. It should be noted that this lemma does not depend on the normality of $E_{0}$.

LEMma 2. Let $t_{0}=x_{1}<t_{1}<\cdots<t_{q+1}=x_{2}$ be a set of values such that there are no pairs of conjugate points on $E_{0}$ relative to $I_{\lambda}$ on any of the intervals $t_{r-1} \leqq x \leqq t_{r},(r=1, \cdots, q+1)$. There exists a qn parameter family of broken isoperimetric extremals

$$
y_{i}\left(x, b_{11}, \cdots, b_{n q}\right), \quad \lambda_{\alpha}\left(b_{11}, \cdots, b_{n q}\right), \quad x_{1} \leqq x \leqq x_{2},
$$

containing $E_{0}$ for $b_{i s}=b_{i s 0}$, satisfying the conditions (2), passing through the points 1,2 , and $\left(x, y_{i}\right)=\left(t_{s}, b_{i s}\right),(s=1, \cdots, q)$, and having no corners on the intervals $t_{r-1}<x<t_{r}$. The functions $y_{i}(x, b), y_{i x}(x, b)$, $\lambda_{\alpha}(b)$ have continuous first and second derivatives for values $(x, b)$ near those belonging to $E_{0}$ except possibly at the corner points. For the arc $E_{b}$ of the family $(7)$ determined by values $(b) \neq\left(b_{0}\right)$ in a sufficiently small neighborhood of $(b)=\left(b_{0}\right)$ one has $J\left(E_{b}\right)>J\left(E_{0}\right)$.

In order to establish this result let

$$
Y_{i}\left(x, b_{11}, \cdots, b_{n q}, \lambda_{1}, \cdots, \lambda_{m}\right), \quad \lambda_{\alpha}, \quad x_{1} \leqq x \leqq x_{2},
$$

* Loc. cit., pp. 253-254.

$\dagger$ The problem of Bolza in the calculus of variations, Annals of Mathematics, (2), vol. 33 (1932), pp. 267-270. 
be a $(q n+m)$ parameter family of broken extremals containing $E_{0}$ for values $b_{i s}=b_{i s 0}, \lambda_{\alpha}=\lambda_{\alpha 0}$, passing through the points 1,2 , and $\left(x, y_{i}\right)=\left(t_{s}, b_{i s}\right)$ and having no corners on the intervals $t_{r-1}<x<t_{r}$. Except at the corner points the functions $Y_{i}(x, b, \lambda), Y_{i x}(x, b, \lambda)$ have continuous first and second derivatives for values $(x, b, \lambda)$ near those belonging to $E_{0}$. The existence of a family of this type follows readily from the first part of Lemma 1 and existence theorems for differential equations of the form (3). When the functions $Y_{i}(x, b, \lambda)$ are substituted in the integrals $(2)$ a set of functions $J_{\alpha}(b, \lambda)$ is obtained having continuous first and second derivatives for values $(b, \lambda)$ near those on $E_{0}$. The functional determinant $\left|\partial J_{\alpha} / \partial \lambda_{\beta}\right|,(\alpha, \beta=1, \cdots, m)$, is different from zero when $(b, \lambda)=\left(b_{0}, \lambda_{0}\right)$, as will be seen in the next paragraph. The equations $J_{\alpha}(b, \lambda)=l_{\alpha}$ are satisfied by the values $(b, \lambda)=\left(b_{0}, \lambda_{0}\right)$ and hence have unique solutions $\lambda_{\alpha}(b)$, with $\lambda_{\alpha}\left(b_{0}\right)$ $=\lambda_{\alpha 0}$, having continuous second derivatives near $(b)=\left(b_{0}\right)$. When the functions $\lambda_{\alpha}(b)$ are substituted for $\lambda_{\alpha}$ in the functions (8), a family (7) is obtained having the properties described in the lemma. The first part of the lemma is immediate. In order to prove the last part we note that by virtue of the identities

$$
y_{i}\left(x_{1}, b\right)=y_{i 1}, \quad y_{i}\left(t_{s}, b\right)=b_{i s}, \quad y_{i}\left(x_{2}, b\right)=y_{i 2},
$$

where $\left(x_{1}, y_{1}\right),\left(x_{2}, y_{2}\right)$ are the points 1 and 2 , respectively, the variations

$$
\delta y_{i}(x)=y_{i b_{j s}}\left(x, b_{0}\right) d b_{j s}, \quad j=1, \cdots, n ; s=1, \cdots, q,
$$

satisfy the relations

$$
\delta y_{i}\left(x_{1}\right)=0, \quad \delta y_{i}\left(t_{s}\right)=d b_{i s}, \quad \delta y_{i}\left(x_{2}\right)=0,
$$

so that $\delta y_{i}(x) \neq 0$ on $x_{1} x_{2}$ if $(d b) \neq(0)$. Moreover the variations $\eta_{i}=\delta y_{i}$ satisfy equations (5). For by construction the value $J_{\alpha}(b)$ of the integral $J_{\alpha}$ along the family $(7)$ is a constant. It follows that $d J_{\alpha}$ $=L_{\alpha}(\delta y)=0$ along $E_{0}$. From the assumptions on $E_{0}$ we have accordingly $Q(\delta y)>0$ whenever $(d b) \neq(0)$. Consider now the function

$$
J_{0}(b)=J(b)+\lambda_{\alpha 0} J_{\alpha}(b)
$$

obtained by evaluating the integral $J_{0}=J+\lambda_{\alpha 0} J_{\alpha}$ along the arc $y_{i}(x, b)$ of the family (7). By the use of the Euler-Lagrange equations (3) and the properties of $\delta y_{i}$ just established, it is found that along $E_{0}$ one has

$$
d J_{0}=\int_{x_{1}}^{x_{2}}\left\{F_{y_{i}} \delta y_{i}+F_{y_{i}} \delta y_{i}^{\prime}\right\} d x=\left.F_{y_{i}} \delta y_{i}\right|_{x_{1}} ^{x_{2}}=0, \quad d^{2} J_{0}=Q(\delta y)>0
$$


for all values $(d b) \neq(0)$. In view of these relations and the equations $J_{\alpha}(b)=l_{\alpha}$ it follows that for values $(b) \neq\left(b_{0}\right)$ near $(b)=\left(b_{0}\right)$ the inequality

$$
0<J_{0}(b)-J_{0}\left(b_{0}\right)=J(b)-J\left(b_{0}\right)
$$

holds, as was to be proved.

The proof of Lemma 2 will be complete if we show that the determinant $\left|\partial J_{\alpha} / \partial \lambda_{\beta}\right|$ is different from zero at $(b, \lambda)=\left(b_{0}, \lambda_{0}\right)$. To prove this let $\delta^{\prime} Y_{i}(x)=Y_{i \lambda_{\beta}}\left(x, b_{0}, \lambda_{0}\right) d \lambda_{\beta}$. For the function $J_{\alpha}(b, \lambda)$ one then has along $E_{0}$

$$
\delta^{\prime} J_{\alpha}=\frac{\partial J_{\alpha}}{\partial \lambda_{\beta}} d \lambda_{\beta}=L_{\alpha}\left(\delta^{\prime} Y\right),
$$

where the functions $L_{\alpha}$ are given by equations (5). From the relations

$$
Y_{i}\left(x_{1}, b, \lambda\right)=y_{i 1}, \quad Y_{i}\left(t_{s}, b, \lambda\right)=b_{i s}, \quad Y_{i}\left(x_{2}, b, \lambda\right)=y_{i 2}
$$

it follows by differentiation that

$$
\delta^{\prime} Y_{i}\left(x_{1}\right)=0, \quad \delta^{\prime} Y_{i}\left(t_{s}\right)=0, \quad \delta^{\prime} Y_{i}\left(x_{2}\right)=0 .
$$

Moreover along $E_{0}$ we have

$$
K_{i}\left(\delta^{\prime} Y\right)+P_{i \beta} d \lambda_{\beta}=0,
$$

where $P_{i \beta}, K_{i}$ are given by equations (4) and (6). This can be seen by substituting the functions (8) in the Euler-Lagrange equations (3), differentiating for $\lambda_{\beta}$, multiplying by $d \lambda_{\beta}$, and summing. Suppose now that the determinant in question were zero. Then there would exist constants $d \lambda_{\beta}$ not all zero such that $\delta^{\prime} J_{\alpha}=0$ along $E_{0}$. If $\delta^{\prime} Y_{i} \equiv 0$ on $x_{1} x_{2}$, one would have $P_{i \beta} d \lambda_{\beta} \equiv 0$ on $x_{1} x_{2}$ by equations (11), and $E_{0}$ could not be normal. Hence $\delta^{\prime} Y_{i} \neq 0$ on $x_{1} x_{2}$. By the use of equations (9), (10), (11), and $\delta^{\prime} J_{\alpha}=0$ and integration by parts it would be found that along $E_{0}$ we would have $L_{\alpha}\left(\delta^{\prime} Y\right)=0$ and

$$
0=\int_{x_{1}}^{x_{2}} \delta^{\prime} Y_{i}\left[K_{i}\left(\delta^{\prime} Y\right)+P_{i \beta} d \lambda_{\beta}\right] d x=Q\left(\delta^{\prime} Y\right)+\delta^{\prime} J_{\beta} d \lambda_{\beta}=Q\left(\delta^{\prime} Y\right),
$$

contrary to our assumption concerning the value of the second variation $Q(\eta)$ along $E_{0}$. Hence $\left|\partial J_{\alpha} / \partial \lambda_{\alpha}\right| \neq 0$ on $E_{0}$ and Lemma 2 is established.

We are now in position to prove Theorem 1 . To do so we let $\mathcal{F}^{\prime}$ be a neighborhood of $E_{0}$ in $x y$-space so small that each subarc of the family (7) in $\mathcal{F}^{\prime}$ with end points on successive hyperplanes $x=t_{r-1}, x=t_{r}$ affords a minimum to the integral $I_{\lambda}$ relative to admissible arcs in $\mathcal{F}^{\prime}$ 
joining its end points. This is possible by virtue of Lemma 1 . Let $\mathcal{f}$ be a second neighborhood of $E_{0}$ interior to $\mathcal{F}^{\prime}$ such that every admissible arc $C$ in $\mathcal{Z}$ joining the points 1 and 2 and satisfying equations (2) cuts the hyperplanes $x=t_{s}$ in points $\left(t_{s}, b_{i s}\right)$ whose $y$-coordinates $b_{i s}$ determine an extremal $E_{b}$ of the family (7) lying in $\mathcal{F}^{\prime}$. By Lemma 1 we have $I_{\lambda}(C) \geqq I_{\lambda}\left(E_{b}\right)$, the multipliers $\lambda_{\alpha}$ being those belonging to $E_{b}$. But since the arcs $C$ and $E_{b}$ satisfy equations (2), this implies that

$$
I_{\lambda}(C)-I_{\lambda}(E)=J(C)-J\left(E_{b}\right) \geqq 0,
$$

the equality holding only in case $C \equiv E_{b}$. Diminish $\mathcal{F}$ if necessary so that $J\left(E_{b}\right) \geqq J\left(E_{0}\right)$, as described in Lemma 2 . We then have $J(C)$ $\geqq J\left(E_{b}\right) \geqq J\left(E_{0}\right)$, the equality holding in both cases only in case $C \equiv E_{0}$. This proves the theorem.

The University of Chicago

\section{A NEW SUMMATION METHOD FOR DIVERGENT SERIES*}

\section{W. A. MERSMAN}

1. Introduction. The method to be given here is a modification of that due to Euler-Knopp. $\dagger$ For the weighted means of the partial sums we use the binomial coefficients, but instead of beginning with the first we begin with the "central" one, that is with the greatest. Thus the initial terms always receive the greatest weight, as in the Cesàro-Hölder method.

In this paper it is shown (1) that this new method includes the first two Cesàro methods, and (2) that it also includes the first EulerKnopp method; further, (3) the exact range of summability of the geometric series is determined. Finally, an example is given which indicates that this method may be more powerful than all those of Cesàro-Hölder, although this statement has not yet been proved.

2. Definitions and notation. Throughout we consider a series $\sum_{k=0}^{\infty} a_{k}$ and denote by $S_{n}$ the sum of its first $n+1$ terms. We define $\sigma_{n}$ as follows:

$$
\sigma_{n}=\frac{1}{4^{n}} \sum_{k=0}^{n} C_{2 n+1, n-k} S_{k},
$$

where $C_{n, k}$ denotes the ordinary binomial coefficient. If $\sigma_{n}$ approaches

* Presented to the Society, April 11, 1936. See abstract 42-5-139.

$\dagger$ K. Knopp, Mathematische Zeitschrift, vol. 15 (1922), pp. 226-253. 